\title{
Beneficial Roles of Microglia and Growth Factors in MS, a Brief Review
}

\author{
Vincent Pons and Serge Rivest* \\ Neuroscience Laboratory, Department of Molecular Medicine, Faculty of Medicine, CHU de Québec Research Center, Laval \\ University, Québec City, QC, Canada
}

Microglia are the brain resident immune cells; they can produce a large variety of growth factors (GFs) to prevent neuronal damages and promote recovery. In neurodegenerative diseases, microglia can play both benefic and deleterious roles, depending on different factors and disease context. In multiple sclerosis, microglia are involved in both demyelination (DM) and remyelination (RM) processes. Recent studies suggest a beneficial role of microglia in regenerative processes. These include the regenerative development of myelin after DM. This review gives an overlook of how microglia and GFs can influence the RM properties.

OPEN ACCESS

Edited by:

Bernhard Theodor Baune,

University of Melbourne, Australia

Reviewed by:

Leyre Mestre,

Cajal Institute (CSIC), Spain

Jelena Skuljec,

Essen University Hospital, Germany

*Correspondence:

Serge Rivest

serge.rivest@crchudequebec.

ulaval.ca

Specialty section:

This article was submitted to

Cellular Neuropathology,

a section of the journal

Frontiers in Cellular Neuroscience

Received: 20 May 2020

Accepted: 10 August 2020

Published: 23 September 2020

Citation:

Pons V and Rivest S (2020) Beneficial Roles of Microglia and Growth Factors in MS, a Brief Review. Front. Cell. Neurosci. 14:284. doi: 10.3389/fncel.2020.00284
Keywords: microglia 1, growth factors, remyelination, multiplesclerosis, innate immnuity

\section{INTRODUCTION}

Microglia belong to the immune system. There is a general consent on the involvement of microglia in neurodegenerative diseases. In multiple sclerosis (MS), microglial cells may have both detrimental and beneficial actions, depending on the circumstances, models, and disease progression and severity. Tissue examination as well as experimental findings show that demyelination (DM) is usually associated with a robust inflammation and the presence of immune cells (Kotter et al., 2005). Microglia are involved in each MS phase; they are one of the main cell types responsible for phagocytic clearance of myelin debris and are able to release a wide range of cytokines and growth factors (GFs) known to contribute to the remyelination (RM; Hinks and Franklin, 1999; Barnett and Prineas, 2004; Glezer et al., 2007; Rivest, 2009). RM is the regenerative process following natural or induced DM occurring in the adult central nervous system (CNS). RM is composed of two major phases. The first consists of the colonization of lesions by oligodendrocyte progenitor cells (OPCs), and the second consists of the differentiation of OPC into mature oligodendrocytes (ODs), which will generate functional myelin sheaths (Chari, 2007). OPCs and their mature counterpart are partially driven by microglia-secreted cytokines and GFs.

This brief review is an overview of the involvement of microglia in RM process through the production of specific GFs.

\section{MICROGLIA}

Microglia are the innate immune cells of CNS. They arise from the yolk sac and invade the newly formed brain in mice between embryonic days 8.5 and 10. These monocytic lineage-derived cells maintain themselves in the brain via self-renewal (Rossi and Lewis, 2018). Microglia represent $5 \%$ to $15 \%$ of adult brain cells (Thion et al., 2018). 
They continuously survey the CNS with their motile processes and are the first responder to insults, such as pathogen infections, toxins, and brain injuries (Shemer et al., 2015; Li and Barres, 2018). Microglia are also known to contribute to CNS development and homeostasis ( $\mathrm{Li}$ and Barres, 2018; Thion et al., 2018) and are involved in neurogenesis and synaptic pruning and also participate to neuronal support and myelogenesis (Nagata, 1997; Paolicelli et al., 2011; Lampron et al., 2015; Shemer et al., 2015). Microglia are highly plastic. They can be polarized and can produce and release a large variety of mediators ranging from cytotoxic such as interleukin $1 \beta$ (IL-1 $\beta$ ), tumor necrosis factor $\alpha$ (TNF- $\alpha$ ), reactive oxygen species, and nitric oxide (NO), IL-4, to trophic factors that provide support to CNS, notably with brain neurotrophic-derived factor (BDNF), insulin like growth factor 1 (IGF-1), arginase-1 (Arg-1), and transforming growth factor $\beta$ (TGF- $\beta$; Ueno et al., 2013). BDNF is a neurotrophic factor, acting on neurons. It supports survival and promotes the growth and differentiation of newly formed neurons and synapses (Flores et al., 2020). IGF-1 inhibits apoptosis and promotes proliferation and differentiation of neural stem cells (Thored et al., 2009). Arg- 1 outcompetes inducible NO synthetase, decreasing the level of NO, thus the inflammation. TGF- $\beta$ acts on inflammation and microglial proliferation. It suppresses the expression of MHC-II, IL-1, and TNF- $\alpha$ (Lee et al., 2017).

The role of microglia under pathologic conditions has been highly studied, and they are believed to be a key therapeutic target in CNS diseases.

\section{A GLOBAL DESCRIPTION OF MS}

MS is an autoimmune and neurodegenerative disease that affects the CNS. The pathology appears between the ages of 20 and 40 years with an autoimmune reaction against components of myelin (Huang et al., 2017). MS affects sensation and motor, autonomic, and neurocognitive function (Sospedra and Martin, 2016). The relapsing-remitting (RR) form is the most common subtype of MS. This form is characterized by acute demyelinating phase, corresponding to an attack on myelin and nerve fibers by immune cells, followed by periods of remission (Ghasemi et al., 2017). Usually, it lasts 5-10 years and evolves in $80 \%$ of cases in secondary chronic-progressive phase (Nazareth et al., 2018). The neuroinflammation and by extension damages in the CNS are due to immune cell activation and their cytokines, resulting in the formation of plaques composed of cells, demyelinated axons, and astrogliosis in the white and gray matter (Compston and Coles, 2008). The inflammation in MS is dominated by $\mathrm{T}$ cells and macrophages, but MS is mainly thought to be a $\mathrm{T}$ cell-mediated autoimmune disease. Antigen-presenting cells following toll-like receptor stimulation produce cytokines, which shape the differentiation of $\mathrm{T}$ cells into a specific subsets (Legroux and Arbour, 2015). $\mathrm{CD}^{+} \mathrm{T}$ cells seem to be the dominant population of T cells (Rangachari et al., 2017), because $\mathrm{CD}^{+} \mathrm{T}$ cells do not exceed $20 \%$ to $30 \%$ of the total T-cell population (Kutzelnigg and Lassmann, 2014). Based on secretion of specific molecules, three main subtypes of $\mathrm{T}$ helper $\left(\mathrm{T}_{\mathrm{H}}\right)$ cells have been described; $\mathrm{T}_{\mathrm{H}} 1$ (interferon- $\gamma$ ), $\mathrm{T}_{\mathrm{H}} 2$ (IL-4, IL5, and IL-13), and $\mathrm{T}_{\mathrm{H}} 17$ (IL-17, IL-21, and IL-22). Noteworthy,
$\mathrm{T}_{\mathrm{H}} 17$ phenotype favors the recruitment of neutrophils and the activation of innate immunity (Raphael et al., 2015). In MS lesion, $\mathrm{CD}^{+}$and $\mathrm{CD}^{+}$express IL-17. $\mathrm{CD}^{+} \mathrm{T}_{\mathrm{H}} 17$ are necessary to develop autoimmune encephalomyelitis (EAE), the main animal model MS (Komiyama et al., 2006). Macrophages and activated microglia are in contact with myelin sheaths, as well as dystrophic axons, and have the ability to phagocyte myelin debris and produce proinflammatory molecules during DM (Brück et al., 1995; Hänninen, 2017).

However, the role of adaptive immunity may not be necessarily harmful. Some studies reveal that neurotrophins such as BDNF are synthesized in active MS plaques (Kutzelnigg and Lassmann, 2014). Moreover, $\mathrm{T}_{\mathrm{H}} 2$ promotes anti-inflammatory response (Ghasemi et al., 2017; Mahallawi et al., 2018).

RM is spontaneous; myelin sheaths are newly formed around axons in CNS following DM. However, RM becomes incomplete or fails with time. This newly formed myelin sheath differs from the original, as it is thinner and shorter (Goldschmidt et al., 2009; Cunniffe and Coles, 2019). The immune system is thought to be harmful in MS, but it is also essential for a proper RM. Indeed, RM is stimulated by the inflammatory process occurring at the DM phase. Myelin debris has an inhibitory effect for OPC differentiation, so its clearance is an important step for RM (Robinson and Miller, 1999; Kotter, 2006; Yong and Rivest, 2009; Cunniffe and Coles, 2019). Chari et al. (2006) have shown that anti-inflammatory molecules can inhibit differentiation of OPCs and delay RM. Other studies have demonstrated a less efficient RM in the absence of MHC-II, inflammatory factors, and active immune system (Arnett et al., 2003). Microglia are deeply involved in MS progression; these cells not only modulate OPC homeostasis, differentiation, maturation, and neurogenesis but also contribute to OD and neuronal death (Pang et al., 2010; Hagemeyer et al., 2017).

\section{ROLE OF MICROGLIA IN RM}

In MS and its animal models, microglia are highly activated and participate in all phases of the disease. Microglial cells show increased expression of inflammatory cytokines and cytotoxic molecules. An accumulating body of evidence supports rather a detrimental role of microglia in neurodegenerative diseases. However, numerous articles indicate that microglia could also exert a protective function in MS and drive the RM process (Lloyd and Miron, 2019). Inefficient microglial activation, phagocytosis, and inflammatory response toward myelin debris during DM process lead to an impaired remyelinating process (Neumann et al., 2008; Lampron et al., 2015; Pons et al., 2020). A change in microglia polarization from activation to repair profile can initiate the RM (Miron et al., 2013). The dual aspect of microglia has been shown in vitro. Lipopolysaccharide (LPS) treatment of microglia has deleterious consequences on neurons, whereas they become neuroprotective when preactivated with IL-4. Conditioned microglia downregulate the release of GFs by TNF- $\alpha$ (Butovsky et al., 2006). Production of GFs by microglia promotes survival and proregenerative activities on ODs and OPCs (Hsieh et al., 2004; Neumann et al., 2008; Voet et al., 2019). IGF-1 protects OPCs from TNF- $\alpha$ and enhances cell 
survival through PI3K/Akt pathway. Furthermore, IGF-1 signals suppress caspase- 9 and caspase- 3 activation, which protects OPCs from the mitochondrial apoptotic pathway (Pang et al., 2007). Concerning DM/RM, a reduced number and area of demyelinating lesions were found in EAE Lewis rats that received systemic IGF-1 injections. Moreover, these lesions contain a higher proportion of proliferative OD cells (Yao et al., 1995; Zeger et al., 2007). However, Cannella et al. (2000) used IGF-1 at different time points during acute and chronic phases of EAE in mice, and they failed to demonstrate a real efficacy of IGF-1 treatment. Their results reveal a transient clinical amelioration and a low RM level, which also did not improve in animals that received IGF-1 at chronic time points (Cannella et al., 2000; Zhang et al., 2011).

A marked increase in BDNF mRNA expression and a slight increase in neurotrophin-4/5 (NT-4/5) levels have been shown in LPS-treated microglia in vitro. These results demonstrate that inflammatory activated microglia have the ability to favor the secretion of GFs and possibly participate in neuronal regeneration and neuroprotection (Var and ByrdJacobs, 2020). The BDNF receptor is found at the site of MS lesion. Interestingly, BDNF is naturally reduced in corpus callosum after cuprizone treatment (VonDran et al., 2011). This neurotrophic factor is detected in macrophages/microglia and $\mathrm{T}$ cells in active and inactive lesions (Kerschensteiner et al., 1999; Kutzelnigg and Lassmann, 2014). Neurons are in distress during MS crisis, and a robust expression of BDNF receptors may compensate to balance the detrimental effects of inflammation (Stadelmann et al., 2002). Furthermore, neutralization of BDNF, NT-3, and nerve growth factor within the cultured tissues enhance MHC class II inducibility (Neumann et al., 1998; Rahimlou et al., 2020). Through this factor, microglia affect the inflammation by impairing the antigenic presentation and then promote the survival of existing neurons and promote neurogenesis and RM (Huang and Reichardt, 2001). BDNF impacts also ODs. Indeed, following cuprizone lesion in mice with a deficiency in BDNF production, VonDran et al. (2011) highlighted a reduced number of NG2-positive cells and deficit in myelin protein but no change in the total numbers of ODs. This suggests that BDNF may regulate the numbers of OPCs following DM. Interestingly, Fletcher et al. (2018) used a BDNF-like molecule, namely, TDP6, which mimics the TrkB-binding region of BDNF, and highlighted the beneficial effect of this molecule to promote myelin regeneration and OD differentiation in mice after a demyelinating insult.

Arginase (Arg-1) is an enzyme that converts arginine to ornithine. In multiple animal models, the internalization of myelin by microglia leads to the expression of Arg-1 (Miron et al., 2013; Guerrero and Sicotte, 2020). In vitro, both microglia and astrocytes produce arginase. Arg-1 activity can reduce the supply of arginine needed for the production of NO (Caldwell et al., 2018). In EAE model, Yang et al. (2016) used spermidine as a treatment, which abolished the expression of inflammatory factors while enhancing the expression of Arg-1 in immune cells and reverses EAE progression. Moreover, macrophages pretreated with Arg-1 inhibitor abrogate the therapeutic effect of spermidine. This study could suggest a partial role of Arg-1 in the disease progression (Yang et al., 2016), but the role of Arg-1 remains controversial. Arg-1 gene is highly up-regulated in the spinal cord of EAE mice. To explore the role of the enzymes, investigators have inhibited Arg-1 in EAE mice, during inductive and effector phases. Impairing Arg-1 altered immune response to myelin OD glycoprotein and improved EAE in mice. NO production is slightly increased, and there is less TNF- $\alpha$ synthesis. It is suggested that Arg-1 could regulate EAE through a NO-independent manner. An impaired immune response can be caused by inhibition of arginase. L-Ornithine, a metabolite from arginase pathway, is necessary for production of polyamine and proline, which control cell proliferation (Xu et al., 2003; Polis et al., 2020). The role of arginase is not clear; further studies are required to better understand the function of this enzyme in MS.

TGF- $\beta$ suppresses inflammatory factors produced by microglia, thereby regulating the inflammatory response by these cells. Moreover, the critical role of this regulatory factor is to maintain tolerance via the regulation of lymphocyte proliferation, differentiation, and survival (Suzumura et al., 1993; Mirshafiey and Mohsenzadegan, 2009). TGF- $\beta$ down-regulates the production of myelin toxic factors such as TNF- $\alpha$ and oxygen radicals and induces a switch of microglia from proinflammatory to become repair cells. A study demonstrates that in the cuprizone model the expression of several trophic factors is up-regulated in the course of DM and RM, namely, TGF- $\beta$ and IGF-1 (Olah et al., 2012; Guerrero and Sicotte, 2020). This overexpression promotes OD differentiation and migration toward lesion site (Hinks and Franklin, 1999; Diemel et al., 2003; Traiffort et al., 2020). Furthermore, Hamaguchi et al. (2019) recently proposed that circulating TGF- $\beta 1$ enters the CNS and could contribute to RM. The study provided evidence that TGF- $\beta 1$ administration promotes RM by stimulating human OD maturation and restore neurological function in MS (Hamaguchi et al., 2019).

Researchers had also a growing interest in macrophagecolony stimulating factor (m-CSF) and its receptor in the past few years. Modulation of the CSF axis could be used as a therapeutic target for many diseases from cancer to neurodegenerative pathologies (Pons and Rivest, 2018). In the brain, microglia are affected by CSF1R activity depending on the context (Pons et al., 2020). Laflamme et al. (2018) have used two different methods to study the role of m-CSF in cuprizone-intoxicated mice. They observed a reduced number of OPCs in the corpus callosum of cuprizone-fed mice bearing a deletion in the CSF1R gene. Moreover, animals exhibited a reduction of the microglial inflammatory response and a higher level of myelin in areas of interest. These data indicate that impairing the m-CSF signaling pathway alters microglial phagocytosis, inflammatory activation, and OPC proliferation. In a second series of experiments, they administered m-CSF during the DM phase. Interestingly, inducing $\mathrm{m}$-CSF signalization restrained myelin loss, boosted microglial activity, increased IGF-1 expression, and promoted the recruitment of OPCs at the lesion site. This suggests that $\mathrm{m}$-CSF has a robust beneficial role in this model and promotes repair by conditioning microglia and OPCs (Laflamme et al., 2018). Despite the promising therapeutic potential, it remains unclear how $\mathrm{m}$-CSF affects microglia. In this 


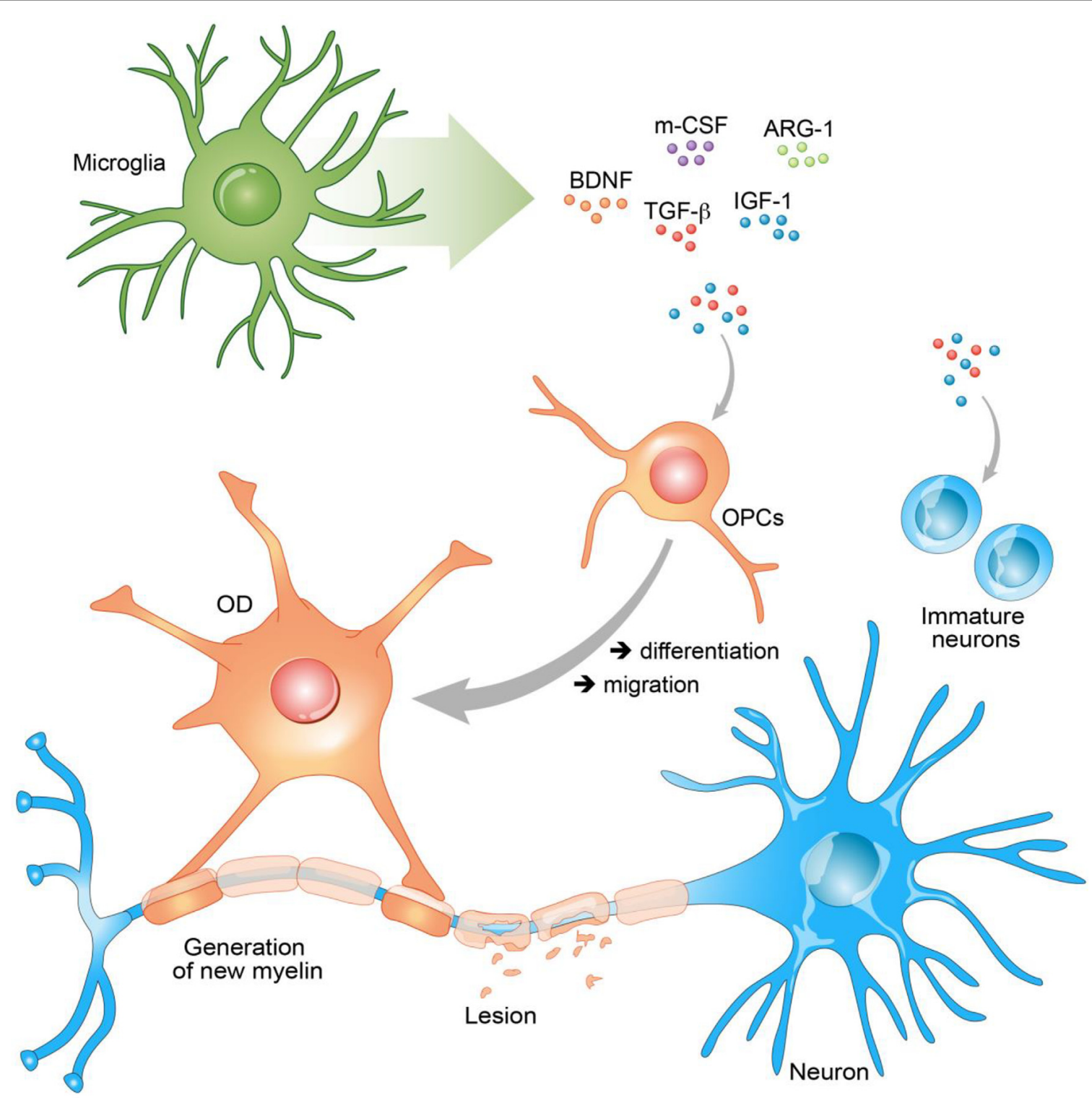

FIGURE 1 | Secreted growth factors (GFs) promote remyelination (RM). Secreted GFs have the ability to stimulate neurons, oligodendrocyte progenitor cells (OPCs), and oligodendrocyte (OD) to promote RM. Brain neurotrophic-derived factor (BDNF), insulin growth factor 1 (IGF-1), arginase-1 (Arg-1), and transforming growth factor $\beta$ (TGF- $\beta$ ) act on the differentiation of OPCs into ODs and their migration to the lesion site. In the same manner through the secretion of these GFs, microglia stimulate immature neurons and promote neurogenesis.

study, authors used a genetic construction specifically located in microglia to suppress the CSF1R gene. In line with these findings, another group that used an m-CSF-deficient mouse also provided evidence that $\mathrm{m}$-CSF deficiency impairs RM process. The lack of this cytokine causes aberrant activation of astrocytes, which may contribute to axonal damages and disturbs RM (Wylot et al., 2019).

Another method to impair CSF1R signaling pathway is to use biochemical molecules, which interfere with a different part of the receptor. However, these molecules bring out different outcomes, depending on experimental settings. Tahmasebi et al. (2019) have shown amelioration of the general condition in cuprizone-fed mice following CSF1R blockade with PLX3397, a molecule that impairs CSF1R signaling. According to these results, a decrease in the microglial inflammatory response is observed in mice treated with PLX3397. The authors reported an increased rate of Olig-2 expression within the corpus callosum and showed that PLX3397 treatment could induce differentiation of OPCs. They hypothesized that the CSF1R inhibitor attenuates inflammation by microglia, and this could lead to a decrease in OPC death rate. These results are quite surprising because many studies have pointed out the beneficial role of inflammation in $\mathrm{RM}$ process and the strong positive action of CSF1R (Kerschensteiner et al., 1999; Arnett et al., 2003; Kotter et al., 
2005; Olah et al., 2012; Miron et al., 2013; Rawji and Yong, 2013; Lampron et al., 2015; Luo et al., 2017; Laflamme et al., 2018). The major difference between chemical and genetic depletion of CSF1R is the specificity. Indeed, molecules are not or at least less specific than genetic ablation. It could have a cross-inhibitory reaction with other receptors from the same family, whereas genetic construction is restricted to a specific cell type. This may explain why results could be different between studies using molecular compounds to inhibit CSF1R.

\section{CONCLUSION}

This review is an overview of the involvement of microglia in RM processes through production of GFs (Figure 1).

The role of microglia in MS is complex and requires extensive studies. However, the last decades provided a good insight on the implication of microglial in DM/RM processes. We have seen the importance of microglia reactivity in DM phase to set the conditions for RM. IGF-1, Arg-1, BDNF, TGF- $\beta$, and $\mathrm{m}$-CSF have a beneficial effect on RM processes. Most of these molecules act on OPCs, while chronic inflammation induced by microglia leads to the death of

\section{REFERENCES}

Arnett, H. A., Wang, Y., Matsushima, G. K., Suzuki, K., and Ting, J. P.-Y. (2003). Functional genomic analysis of remyelination reveals importance of inflammation in oligodendrocyte regeneration. J. Neurosci. 23, 9824-9832. doi: 10.1523/jneurosci.23-30-09824.2003

Barnett, M. H., and Prineas, J. W. (2004). Relapsing and remitting multiple sclerosis: pathology of the newly forming lesion. Ann. Neurol. 55, 458-468. doi: 10.1002/ana.20016

Brück, W., Porada, P., Poser, S., Rieckmann, P., Hanefeld, F., Kretzschmarch, H. A., et al. (1995). Monocyte/macrophage differentiation in early multiple sclerosis lesions: macrophages in MS. Ann. Neurol. 38, 788-796. doi: 10.1002/ana.410380514

Butovsky, O., Ziv, Y., Schwartz, A., Landa, G., Talpalar, A. E., Pluchino, S., et al. (2006). Microglia activated by IL- 4 or IFN- $\gamma$ differentially induce neurogenesis and oligodendrogenesis from adult stem/progenitor cells. Mol. Cell. Neurosci. 31, 149-160. doi: 10.1016/j.mcn.2005.10.006

Caldwell, R. W., Rodriguez, P. C., Toque, H. A., Narayanan, S. P., and Caldwell, R. B. (2018). Arginase: a multifaceted enzyme important in health and disease. Physiol. Rev. 98, 641-665. doi: 10.1152/physrev.00037.2016

Cannella, B., Pitt, D., Capello, E., and Raine, C. S. (2000). Insulin-Like growth factor-1 fails to enhance central nervous system myelin repair during autoimmune demyelination. Am. J. Pathol. 157, 933-943. doi: 10.1016/s00029440(10)64606-8

Chari, D. M. (2007). Remyelination in multiple sclerosis. Int. Rev. Neurobiol. 79, 589-620. doi: 10.1016/S0074-7742(07)79026-8

Chari, D. M., Zhao, C., Kotter, M. R., Blakemore, W. F., and Franklin, R. J. M. (2006). Corticosteroids delay remyelination of experimental demyelination in the rodent central nervous system. J. Neurosci. Res. 83, 594-605. doi: 10.1002/jnr.20763

Compston, A., and Coles, A. (2008). Multiple sclerosis. Lancet 372, 1502-1517. doi: 10.1016/S0140-6736(08)61620-7

Cunniffe, N., and Coles, A. (2019). Promoting remyelination in multiple sclerosis. J. Neurol. doi: 10.1007/s00415-019-09421-x [Epub ahead of print].

Diemel, L. T., Jackson, S. J., and Cuzner, M. L. (2003). Role for TGF- $\beta 1$, FGF-2 and PDGF-AA in a myelination of CNS aggregate cultures enriched with macrophages. J. Neurosci. Res. 74, 858-867. doi: 10.1002/jnr.10837

Fletcher, J. L., Wood, R. J., Nguyen, J., Norman, E. M. L., Jun, C. M. K., Prawdiuk, A. R., et al. (2018). Targeting TrkB with a brain-derived
ODs. As a central player, microglia are really attractive targets in neurodegenerative diseases, including Alzheimer disease and MS. Dysregulation of microglial function, activation, and secretion of GFs remains poorly understood. It is critical to fully comprehend the role and action of microglia to focus on new efficient therapies. The modulation of these GFs influences the differentiation and proliferation of OPCs. These results suggest that the modulation of microglia or OPCs in diseases may represent an interesting therapeutic strategy to promote RM.

\section{AUTHOR CONTRIBUTIONS}

\author{
VP and SR wrote the manuscript together.
}

\section{FUNDING}

This work was supported by the Canadian Institutes in Health Research (CIHR no. 143279, Foundation Scheme Program) and les Fonds de recherche du Québec, Santé (FRQS) via the research center funding grant. SR is supported by a Canada Research Chair in Neuroimmunology.

neurotrophic factor mimetic promotes myelin repair in the brain. J. Neurosci. 38, 7088-7099. doi: 10.1523/jneurosci.0487-18.2018

Flores, K. R., Viccaro, F., Aquilini, M., Scarpino, S., Ronchetti, F., Mancini, R., et al. (2020). Protective role of brain derived neurotrophic factor (BDNF) in obstructive sleep apnea syndrome (OSAS) patients. Edited by Claudio Tripodo. PLoS One 15:e227834. doi: 10.1371/journal.pone. 0227834

Ghasemi, N., Razavi, S., and Nikzad, E. (2017). Multiple sclerosis: pathogenesis, symptoms, diagnoses and cell-based therapy. Cell J. 19, 1-10. doi: 10.22074/cellj.2016.4867

Glezer, I., Simard, A. R., and Rivest, S. (2007). Neuroprotective role of the innate immune system by microglia. Neuroscience 147, 867-883. doi: 10.1016/j. neuroscience.2007.02.055

Goldschmidt, T., Antel, J., Konig, F. B., Bruck, W., and Kuhlmann, T. (2009). Remyelination capacity of the MS brain decreases with disease chronicity. Neurology 72, 1914-1921. doi: 10.1212/WNL.0b013e3181a8260a

Guerrero, B. L., and Sicotte, N. L. (2020). Microglia in multiple sclerosis: friend or foe? Front. Immunol. 11:374. doi: 10.3389/fimmu.2020.00374

Hagemeyer, N., Hanft, K.-M., Akriditou, M.-A., Unger, N., Park, E. S., Stanley, E. R., et al. (2017). Microglia contribute to normal myelinogenesis and to oligodendrocyte progenitor maintenance during adulthood. Acta Neuropathol. 134, 441-458. doi: 10.1007/s00401-017-1747-1

Hamaguchi, M., Muramatsu, R., Fujimura, H., Mochizuki, H., Kataoka, H., and Yamashita, T. (2019). Circulating transforming growth factor$\beta 1$ facilitates remyelination in the adult central nervous system. eLife 8:e41869. doi: 10.7554/eLife.41869

Hänninen, A. (2017). Infections in MS: an innate immunity perspective. Acta Neurol. Scand. 136, 10-14. doi: 10.1111/ane.12838

Hinks, G. L., and Franklin, R. J. M. (1999). Distinctive patterns of PDGFA, FGF-2, IGF-I and TGF- $\beta 1$ gene expression during remyelination of experimentally-induced spinal cord demyelination. Mol. Cell. Neurosci. 14, 153-168. doi: 10.1006/mcne.1999.0771

Hsieh, J., Aimone, J. B., Kaspar, B. K., Kuwabara, T., Nakashima, K., and Gage, F. H. (2004). IGF-I instructs multipotent adult neural progenitor cells to become oligodendrocytes. J. Cell Biol. 164, 111-122. doi: 10.1083/jcb. 200308101

Huang, W.-J., Chen, W.-W., and Zhang, X. (2017). Multiple sclerosis: pathology, diagnosis and treatments. Exp. Ther. Med. 13, 3163-3166. doi: 10.3892/etm. 2017.4410 
Huang, E. J., and Reichardt, L. F. (2001). Neurotrophins: roles in neuronal development and function. Ann. Rev. Neurosci. 24, 677-736. doi: 10.1146/annurev.neuro.24.1.677

Kerschensteiner, M., Gallmeier, E., Behrens, L., Leal, V. V., Misgeld, T., Klinkert, W. E. F., et al. (1999). Activated human T cells, B cells and monocytes produce brain-derived neurotrophic factor in vitro and in inflammatory brain lesions: a neuroprotective role of inflammation? J. Exp. Med. 189, 865-870. doi: 10.1084/jem.189.5.865

Komiyama, Y., Nakae, S., Matsuki, T., Nambu, A., Ishigame, H., Kakuta, S., et al. (2006). IL-17 plays an important role in the development of experimental autoimmune encephalomyelitis. J. Immunol. 177, 566-573. doi: 10.4049/jimmunol.177.1.566

Kotter, M. R. (2006). Myelin impairs CNS remyelination by inhibiting oligodendrocyte precursor cell differentiation. J. Neurosci. 26, 328-332. doi: 10.1523/jneurosci.2615-05.2006

Kotter, M. R., Zhao, C., van Rooijen, N., and Franklin, R. J. M. (2005). Macrophage-depletion induced impairment of experimental CNS remyelination is associated with a reduced oligodendrocyte progenitor cell response and altered growth factor expression. Neurobiol. Dis. 18, 166-175. doi: 10.1016/j.nbd.2004.09.019

Kutzelnigg, A., and Lassmann, H. (2014). Pathology of multiple sclerosis and related inflammatory demyelinating diseases. Handb. Clin. Neurol. 122, 15-58. doi: 10.1016/B978-0-444-52001-2.00002-9

Laflamme, N., Cisbani, G., Préfontaine, P., Srour, Y., Bernier, J., St-Pierre, M.K., et al. (2018). MCSF-induced microglial activation prevents myelin loss and promotes its repair in a mouse model of multiple sclerosis. Front. Cell. Neurosci. 12:178. doi: 10.3389/fncel.2018.00178

Lampron, A., Larochelle, A., Laflamme, N., Préfontaine, P., Plante, M.-M., Sánchez, M. G., et al. (2015). Inefficient clearance of myelin debris by microglia impairs remyelinating processes. J. Exp. Med. 212, 481-495. doi: 10.1084/jem. 20141656

Lee, P. W., Severin, M. E., and Lovett-Racke, A. E. (2017). TGF- $\beta$ regulation of encephalitogenic and regulatory T cells in multiple sclerosis. Eur. J. Immunol. 47, 446-453. doi: 10.1002/eji.201646716

Legroux, L., and Arbour, N. (2015). Multiple sclerosis and T-lymphocytes: an entangled story. J. Neuroimmune Pharmacol. 10, 528-546. doi: 10.1007/s11481015-9614-0

Li, Q., and Barres, B. A. (2018). Microglia and macrophages in brain homeostasis and disease. Nat. Rev. Immunol. 18, 225-242. doi: 10.1038/nri.2017.125

Lloyd, A. F., and Miron, V. E. (2019). The pro-remyelination properties of microglia in the central nervous system. Nat. Rev. Neurol. 15, 447-458. doi: 10.1038/s41582-019-0184-2

Luo, C., Jian, C., Liao, Y., Huang, Q., Wu, Y., Liu, X., et al. (2017). The role of microglia in multiple sclerosis. Neuropsychiatr. Dis. Treat. 13, 1661-1667. doi: 10.2147/NDT.S140634

Mahallawi, W. H., Khabour, O. F., Zhang, Q., Makhdoum, H. M., and Suliman, B. A. (2018). MERS-CoV infection in humans is associated with a pro-inflammatory Th1 and Th17 cytokine profile. Cytokine 104, 8-13. doi: 10.1016/j.cyto.2018.01.025

Miron, V. E., Boyd, A., Zhao, J.-W., Yuen, T. J., Ruckh, J. M., Shadrach, J. L., et al. (2013). M2 microglia and macrophages drive oligodendrocyte differentiation during CNS remyelination. Nat. Neurosci. 16, 1211-1218. doi: 10.1038/ nn.3469

Mirshafiey, A., and Mohsenzadegan, M. (2009). TGF- $\beta$ as a promising option in the treatment of multiple sclerosis. Neuropharmacology 56, 929-936. doi: 10.1016/j.neuropharm.2009.02.007

Nagata, S. (1997). Apoptosis by death factor. Cell 88, 355-365. doi: 10.1016/s00928674(00)81874-7

Nazareth, T. A., Rava, A. R., Polyakov, J. L., Banfe, E. N., Waltrip, R. W. II., Zerkowski, K. B., et al. (2018). Relapse prevalence, symptoms and health care engagement: patient insights from the multiple sclerosis in America 2017 survey. Mult. Scler. Relat. Disord. 26, 219-234. doi: 10.1016/j.msard.2018. 09.002

Neumann, H., Kotter, M. R., and Franklin, R. J. M. (2008). Debris clearance by microglia: an essential link between degeneration and regeneration. Brain 132, 288-295. doi: 10.1093/brain/awn109

Neumann, H., Misgeld, T., Matsumuro, K., and Wekerle, H. (1998). Neurotrophins inhibit major histocompatibility class II inducibility of microglia: involvement of the P75 neurotrophin receptor. Proc. Natl. Acad. Sci. U S A 95, 5779-5784. doi: 10.1073/pnas.95.10.5779

Olah, M., Amor, S., Brouwer, N., Vinet, J., Eggen, B., Biber, K., et al. (2012). Identification of a microglia phenotype supportive of remyelination. Glia 60, 306-321. doi: 10.1002/glia.21266

Pang, Y., Campbell, L., Zheng, B., Fan, L., Cai, Z., and Rhodes, P. (2010). Lipopolysaccharide-activated microglia induce death of oligodendrocyte progenitor cells and impede their development. Neuroscience 166, 464-475. doi: 10.1016/j.neuroscience.2009.12.040

Pang, Y., Zheng, B., Fan, L.-W., Rhodes, P. G., and Cai, Z. (2007). IGF-1 protects oligodendrocyte progenitors against $\mathrm{TNF} \alpha$-induced damage by activation of PI3K/Akt and interruption of the mitochondrial apoptotic pathway. Glia 55, 1099-1107. doi: 10.1002/glia.20530

Paolicelli, R. C., Bolasco, G., Pagani, F., Maggi, L., Scianni, M., Panzanelli, P., et al. (2011). Synaptic pruning by microglia is necessary for normal brain development. Science 333, 1456-1458. doi: 10.1126/science.12 02529

Polis, B., Srikanth, K. D., Gurevich, V., Bloch, N., Gil-Henn, H., and Samson, A. O. (2020). Arginase inhibition supports survival and differentiation of neuronal precursors in adult Alzheimer's disease mice. Int. J. Mol. Sci. 21:1133. doi: 10.3390/ijms21031133

Pons, V., Laflamme, N., Préfontaine, P., and Rivest, S. (2020). Role of macrophage colony-stimulating factor receptor on the proliferation and survival of microglia following systemic nerve and cuprizone-induced injuries. Front. Immunol. 11:47. doi: 10.3389/fimmu.2020.00047

Pons, V., and Rivest, S. (2018). New therapeutic avenues of MCSF for brain diseases and injuries. Front. Cell. Neurosci. 12:499. doi: 10.3389/fncel.2018. 00499

Rahimlou, M., Hosseini, S. A., Majdinasab, N., Haghighizadeh, M. H., and Husain, D. (2020). Effects of long-term administration of multi-strain probiotic on circulating levels of BDNF, NGF, IL-6 and mental health in patients with multiple sclerosis: a randomized, double-blind, placebo-controlled trial. Nutr. Neurosci. doi: 10.1080/1028415x.2020.1758887 [Epub ahead of print].

Rangachari, M., Kerfoot, S. M., Arbour, N., and Alvarez, J. I. (2017). Editorial: lymphocytes in MS and EAE: more than just a CD4 $4^{+}$World. Front. Immunol. 8:133. doi: 10.3389/fimmu.2017.00133

Raphael, I., Nalawade, S., Eagar, T. N., and Forsthuber, T. G. (2015). T cell subsets and their signature cytokines in autoimmune and inflammatory diseases. Cytokine 74, 5-17. doi: 10.1016/j.cyto.2014.09.011

Rawji, K. S., and Yong, V. W. (2013). The benefits and detriments of macrophages/microglia in models of multiple sclerosis. Clin. Dev. Immunol. 2013:948976. doi: 10.1155/2013/948976

Rivest, S. (2009). Regulation of innate immune responses in the brain. Nat. Rev. Immunol. 9, 429-439. doi: 10.1038/nri2565

Robinson, S., and Miller, R. H. (1999). Contact with central nervous system myelin inhibits oligodendrocyte progenitor maturation. Dev. Biol. 216, 359-368. doi: 10.1006/dbio.1999.9466

Rossi, F., and Lewis, C. (2018). Microglia's heretical self-renewal. Nat. Neurosci. 21, 455-456. doi: 10.1038/s41593-018-0123-3

Shemer, A., Erny, D., Jung, S., and Prinz, M. (2015). Microglia plasticity during health and disease: an immunological perspective. Trends Immunol. 36, 614-624. doi: 10.1016/j.it.2015.08.003

Sospedra, M., and Martin, R. (2016). Immunology of multiple sclerosis. Semin. Neurol. 36, 115-127. doi: 10.1055/s-0036-1579739

Stadelmann, C., Kerschensteiner, M., Misgeld, T., Brück, W., Hohlfeld, R., and Lassmann, H. (2002). BDNF and Gp145trkB in multiple sclerosis brain lesions: neuroprotective interactions between immune and neuronal cells? Brain 125 , 75-85. doi: 10.1093/brain/awf015

Suzumura, A., Sawada, M., Yamamoto, H., and Marunouchi, T. (1993). Transforming growth factor-beta suppresses activation and proliferation of microglia in vitro. J. Immunol. 151, 2150-2158.

Tahmasebi, F., Pasbakhsh, P., Mortezaee, K., Madadi, S., Barati, S., and Kashani, I. R. (2019). Effect of the CSF1R inhibitor PLX3397 on remyelination of corpus callosum in a cuprizone-induced demyelination mouse model. J. Cell. Biochem. 120, 10576-10586. doi: 10.1002/jcb.28344

Thion, M. S., Ginhoux, F., and Garel, S. (2018). Microglia and early brain development: an intimate journey. Science 362, 185-189. doi: 10.1126/science. aat0474 
Thored, P., Heldmann, U., Gomes-Leal, W., Gisler, R., Darsalia, V., Taneera, J., et al. (2009). Long-term accumulation of microglia with proneurogenic phenotype concomitant with persistent neurogenesis in adult subventricular zone after stroke. Glia 57, 835-849. doi: 10.1002/glia.20810

Traiffort, E., Kassoussi, A., Zahaf, A., and Laouarem, Y. (2020). Astrocytes and microglia as major players of myelin production in normal and pathological conditions. Front. Cell. Neurosci. 14:79. doi: 10.3389/fncel.2020.00079

Ueno, M., Fujita, Y., Tanaka, T., Nakamura, Y., Kikuta, J., Ishii, M., et al. (2013). Layer V cortical neurons require microglial support for survival during postnatal development. Nat. Neurosci. 16, 543-551. doi: 10.1038/nn.3358

Var, S. R., and Byrd-Jacobs, C. A. (2020). Role of macrophages and microglia in zebrafish regeneration. Int. J. Mol. Sci. 21:4768. doi: 10.3390/ijms211 34768

Voet, S., Prinz, M., and van Loo, G. (2019). Microglia in central nervous system inflammation and multiple sclerosis pathology. Trends Mol. Med. 25, 112-123. doi: 10.1016/j.molmed.2018.11.005

VonDran, M. W., Singh, H., Honeywell, J. Z., and Dreyfus, C. F. (2011). Levels of BDNF impact oligodendrocyte lineage cells following a cuprizone lesion. J. Neurosci. 31, 14182-14190. doi: 10.1523/jneurosci.6595-10.2011

Wylot, B., Mieczkowski, J., Niedziolka, S., Kaminska, B., and Zawadzka, M. (2019). CSF1 deficiency dysregulates glial responses to demyelination and disturbs CNS white matter remyelination. Cells 9:99. doi: 10.3390/cells9010099

Xu, L., Hilliard, B., Carmody, R. J., Tsabary, G., Shin, H., Christianson, D. W., et al. (2003). Arginase and autoimmune inflammation in the central nervous system. Immunology 110, 141-148. doi: 10.1046/j.1365-2567.2003.01713.x

Yang, Q., Zheng, C., Cao, J., Cao, G., Shou, P., Lin, L., et al. (2016). Spermidine alleviates experimental autoimmune encephalomyelitis through inducing inhibitory macrophages. Cell Death Differ. 23, 1850-1861. doi: 10.1038/cdd. 2016.71

Yao, D. L., Liu, X., Hudson, L. D., and Webster, H. D. (1995). Insulin-like growth factor I treatment reduces demyelination and up-regulates gene expression of myelin-related proteins in experimental autoimmune encephalomyelitis. Proc. Natl. Acad. Sci. U S A 92, 6190-6194. doi: 10.1073/pnas.92.13.6190

Yong, V. W., and Rivest, S. (2009). Taking advantage of the systemic immune system to cure brain diseases. Neuron 64, 55-60. doi: 10.1016/j.neuron.2009. 09.035

Zeger, M., Popken, G., Zhang, J., Xuan, S., Lu, Q. R., Schwab, M. H., et al. (2007). Insulin-like growth factor type 1 receptor signaling in the cells of oligodendrocyte lineage is required for normal in vivo oligodendrocyte development and myelination. Glia 55, 400-411. doi: 10.1002/glia.20469

Zhang, J., Kramer, E. G., Mahase, S., Dutta, D. J., Bonnamain, V., Argaw, A. T., et al. (2011). Targeting oligodendrocyte protection and remyelination in multiple sclerosis. Mt. Sinai J. Med. 78, 244-257. doi: 10.1002/msj.20244

Conflict of Interest: The authors declare that the research was conducted in the absence of any commercial or financial relationships that could be construed as a potential conflict of interest.

Copyright (C) 2020 Pons and Rivest. This is an open-access article distributed under the terms of the Creative Commons Attribution License (CC BY). The use, distribution or reproduction in other forums is permitted, provided the original author(s) and the copyright owner(s) are credited and that the original publication in this journal is cited, in accordance with accepted academic practice. No use, distribution or reproduction is permitted which does not comply with these terms. 